\title{
MD simulations of loading rate dependence of detwinning deformation in nanocrystalline $\mathrm{Ni}$
}

\author{
SU Hao \& TANG QiHeng* \\ State Key Laboratory of Nonlinear Mechanics, Institute of Mechanics, Chinese Academy of Sciences, Beijing 100190, China
}

Received November 26, 2012; accepted January 5, 2013

\begin{abstract}
Molecular dynamics simulations are performed to investigate the deformation behavior of nanocrystalline Ni with pre-twin atom structure. The simulation sample is composed of four grains with average size $12 \mathrm{~nm}$. The simulation technique of isobaric-isothermal ensemble (NPT) with high pressure is applied to obtain a sample with two circle twins. Under uniaxial tensile and shear loading, as well as different detwinning deformation behaviors are observed. Under uniaxial tension the detwinning deformation is induced by the event of grain growth, and it is supported by local energy analysis. Under the shear loading the detwinning deformation is related to the loading rate. The results show that there may be a critical shear rate. As the shear rate is sufficiently high the circle twin is found to be failed; as the shear rate is less than that rate, the size of circle twin become smaller and gradually approach a constant value. Our simulation results are in good agreement with experiment observation.
\end{abstract}

molecular dynamics simulation, detwinning, loading rate

PACS number(s): 61.46.HK, 61.72Bb, 61.72.Mn, 47.11.Mn

Citation: $\quad$ Su H, Tang Q H. MD simulations of loading rate dependence of detwinning deformation in nanocrystalline Ni. Sci China-Phys Mech Astron, 2013, 56: 491-497, doi: 10.1007/s11433-013-5010-z

Nanocrystalline metals have been the subject of considerable research in recent years, with progress made in the processing of materials [1,2] and in computational materials science [3-5]. Some advantages of nanotwinned (nt) materials in comparison with nanomaterials have been reported [1]. It is known that nanometer metals have high strength but with low ductility because of early necking [6] which hinders their application in engineering. Nanotwinned metals have been demonstrated to have a strength comparable to that of nanocrystalline (nc) metals and a considerably higher ductility [2].

Hall-Petch plots of the yield strength of $\mathrm{nc} \mathrm{Cu}$ vs. grain size have been understood by dislocation activities and grain boundary deformation mechanism. Similar Hall-Petch relation of the yield strength of $\mathrm{nt} \mathrm{Cu}$ versus nt spacing has been analyzed. Investigation given by $\mathrm{Li}$ et al. [7] revealed

*Corresponding author (email: qhtang@imech.ac.cn) that in nano-twinned copper the strength depends on the grain size and twin-boundary spacing, the smaller the grain size, the smaller the critical twin-boundary spacing, and the higher the maximum strength. They also showed that if there are dislocation nucleation sites as well. This dislocation motion is not confined, however dislocation nucleation may govern the strength of such material. Both experimental investigations and atomistic simulations reveal that nano-twinned metals possess ultrahigh strength and ductility [8-10]. Twinning is a major deformation mode in metals [11], its effects on material deformation is accompanied by dislocation activities. Wu et al. [12] observed a deformation mechanism, partial dislocation emission from grain boundary in $\mathrm{nc} \mathrm{Al}$ powder, which consequently resulted in deformation stacking faults and twinning. Froseth et al. [13] performed the atomic-level simulations of $\mathrm{nc} \mathrm{Al}$, and found that the presence of grown-in twins may enhance plastic deformation via twin-migration and partial dislocation ac- 
tivity. Their results are discussed in terms of generalized planar fault energy curves. Ogata et al. [14] calculated the energy landscapes of deformation twinning $\mathrm{Mo}, \mathrm{Al}$ and $\mathrm{Cu}$ by using density functional theory. The results show that the twin boundary migration energy is much less than that of the twin boundary formation energy. Thus it is suggested that twin boundary migration is more feasible in comparison to twin boundary formation.

Recently detwinning process (to annihilate twin) has been observed to occur during the deformation of nanocrystalline [15-18]. Twinning and detwinning are two important deformation processes in nc materials. They compete with each other and have important effects on the mechanical behaviors of nc or nt materials [18]. There is no clear understanding on the conditions required for these individual processes to be activated and to predominate.

The experimental observations given by $\mathrm{Ni}$ et al. [19] and Hodge et al. [20] revealed that twinning and detwinning processes are related to grain sizes. The twinning process dominates in a certain range of grain sizes, whereas, the detwinning process dominates outside of this range to annihilate twins. Their experimental observations establish a full spectrum of grain sizes. In the torsion experiments, twin stability is highly correlated to the role of twin boundaries. Even as the shear strain reaches 1 , the persistent twinning in the grain is observed.

In this paper, molecular dynamics (MD) simulation is applied to study the detwinning deformation of nc Ni by both uniaxial tensile and the shear loading. Special attention is paid to the comparison of the different detwinning deformation mechanisms. Twin evolution companied by dislocation activities is observed, and the energy analysis is given.

\section{Computation and modeling}

\subsection{Interatomic potential}

It is known that the accuracy of MD simulations is dictated by the accuracy of interatomic potential being used. The embedded atom method (EAM) to describe potential for face cubic center (FCC) Ni [21] is adopted as thus:

$$
E_{T}=\sum_{i=1}^{N} F_{i}\left(\rho_{i}\right)+\frac{1}{2} \sum_{i j, i \neq j} \varphi_{i j}\left(r_{i j}\right),
$$

where $\varphi_{i j}\left(r_{i j}\right)$ is the pair interaction and $r_{i j}$ is the distance between atoms $i$ and $j, F_{i}$ is the embedding function and $\rho_{i}$ is the electronic density at the site of atom i. Most simulation results show that the EAM potential is valid for metallic materials. All parameters for the interatomic potential of $\mathrm{Ni}$ can be found [21].

The components of the stress tensor associated with an atom are defined as [22]:

$$
\sigma_{k l}=\frac{1}{\Omega_{0}}\left(-\frac{p_{k} p_{l}}{m}+\sum_{j \neq i} \frac{1}{2} \frac{\partial \varphi}{\partial r} \frac{r_{i j}^{k} r_{i j}^{l}}{r_{i j}}\right),
$$

where $\sigma_{k l}$ is the stress component, $p$ is the atomic momentum, $\mathrm{m}$ is the atomic mass, $r_{i j}^{k}$ and $r_{i j}^{l}$ are the k-th, 1-th components of $r_{i j}$, and $\Omega_{0}$ is the volume of an atom.

\subsection{Models of nanocrystalline Ni}

The initial simulated nc contains four grains named as $\mathrm{G} 1$, G2, G3 and G4, and four grain boundaries GB1, GB2, GB3 and GB4. A NPT simulation technique with -4 GPa pressure at room temperature is carried out to relax any possible high potential energy configuration, with the Parrinello-Rahman approach [23] applied to periodic boundary conditions. The crystal orientation of $\mathrm{G} 1$ is along $x[111], y[1-10]$ and $z$ [11-2]. Grains G2, G3 and G4 are obtained by rotating G1 in $\mathrm{z}$ axis with $60^{\circ}, 90^{\circ}$, and $-60^{\circ}$ respectively. The sample size is with $68 a_{0} \times 100 a_{0} \times \sqrt{6} a_{0}$ about 67000 atoms, $\mathrm{a}_{0}$ is a crystal constant $(3.52 \AA)$. Figure $1 \mathrm{a}$ and $1 \mathrm{~b}$ display atomic configurations before NPT simulation and after NPT simulation, respectively. After NPT relaxation, some stacking faults at the different grains are observed, and two twins with the circle structure are constructed at G2 and G4, respectively.

The isobaric-isothermal ensemble (NPT) is frequently used to simulate the mechanical behavior of nanometer materials [24-26]. The method of constant-NPT with high pressure is similar to the experimental technique of inert gas condensation [27] which is primarily used to prepare nanometer materials [28] in nanometer engineering. The simulation sample with twin structure in this paper is naturally obtained in the process of NPT simulation, and the twin structure displayed as a circle. In contrast, the twin structure is synthesized and embedded in the grains [7,29], the artificial twin structure being a straight line.

\subsection{Computational method}

The atom configuration at Figure $1 \mathrm{~b}$ with the twin is selected as the initial model. Initial velocities of atoms are specified based on the Maxwellian distribution corresponding to $T=300 \mathrm{~K}$, and the magnitudes may be adjusted so as to keep temperature constant in the system according to the Nose-Hoover thermostat [30,31]. The time integration of motion is performed by using the velocity-Verlet algorithm [32].

In order to understand well the deformation mechanism of $\mathrm{nc} \mathrm{Ni}$, a common neighbor analysis technique [33,34] is adopted to measure the local crystal structure of a particular atom. Colors are assigned to atoms, red for the perfect FCC atoms, and green for the HCP atoms, and blue for the NON (or amorphous) atoms. Two adjacent green lines represent a 

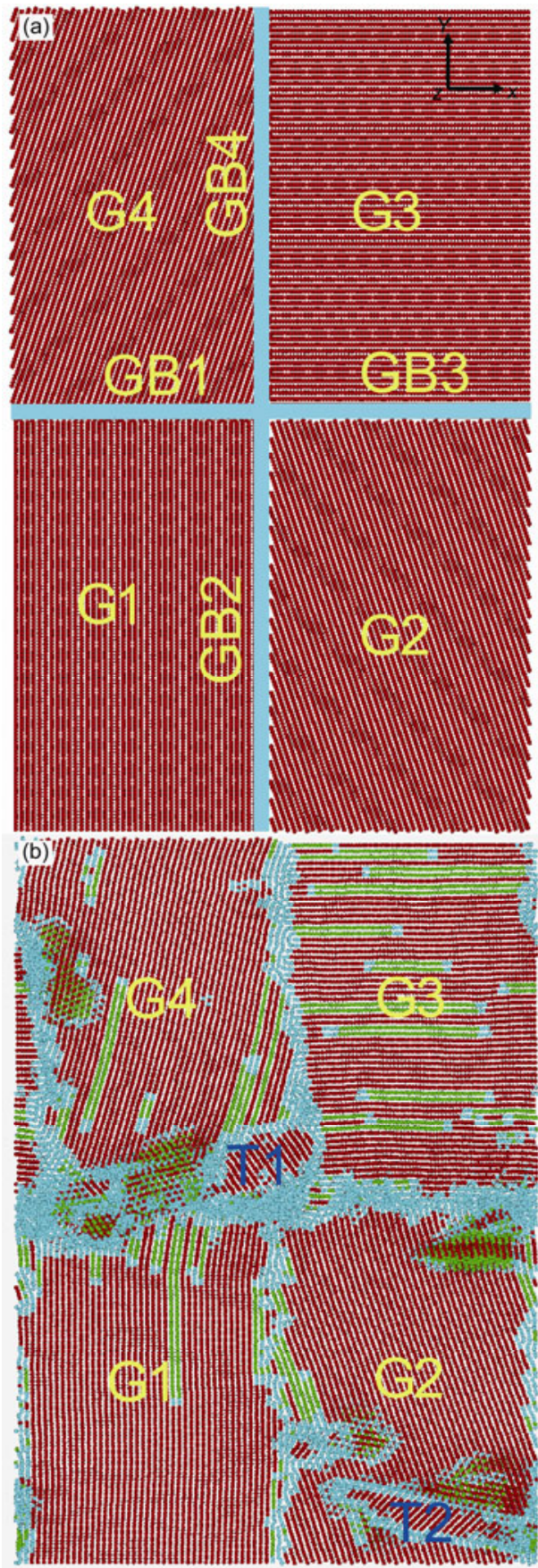

Figure 1 (a) Schematic of nanocrystalline Ni model before NPT simulation; (b) atomic configuration after NPT simulation.

stacking fault [35].

\section{Results and discussion}

\subsection{Uniaxial tensile}

The sample of nc-Ni is loaded in uniaxial tensile. The loading strain rate is $5 \times 10^{8} \mathrm{~s}^{-1}$. The simulation results are shown in Figure 2, the interface GB1 between G1 and G4 disap-
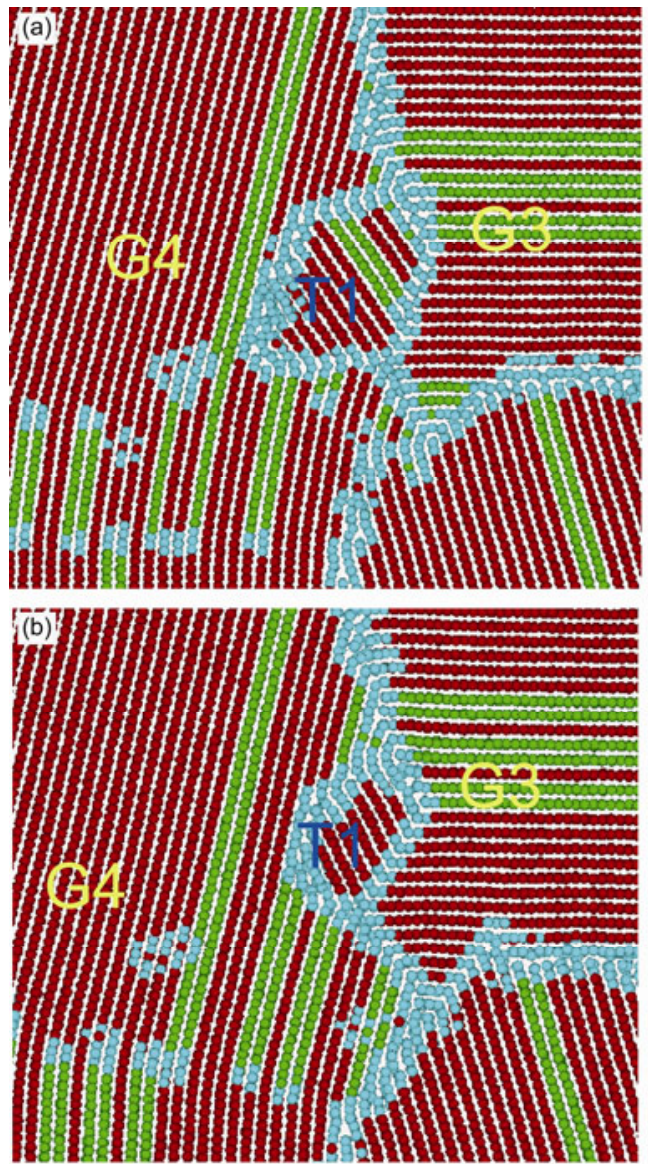

Figure 2 Atomic configurations of grain 1 and 4 during the tensile deformation. (a) $\varepsilon_{y y}=2.5 \%$; (b) $\varepsilon_{y y}=5.0 \%$.

pears gradually with increment deformation. Near the triple junction region of G1, G3 and G4, there is a small twin structure termed as $\mathrm{T} 1$ and this twin structure is stable during the initial stage of tensile deformation. Compared Figures 2 (a) with 2 (b), as strain is $2.5 \%$ and $5 \%$, the size of twin T1 reduces but it keeps its circle structure. During the deformation process the number of amorphous atoms near GB1 region reduces because of recrystallization of G1 and G4. As strain is reached to $2.5 \%$, GB1 disappears by the rotation deformation of grains G1 and G4. The phenomena of grain rotation and recrystallization are frequently observed in metal deformation. Using low energy electron diffraction (LEED) technique, Farnsworth et al. [36] observed recrystallization at high temperature heat treatment of metals Mo, W , Ta and Re. Huang et al. [37] applied a numerical simulation technique to study the recrystallization of the hexagonal close-packed (HCP) metal crystal of titanium at elevated temperature, the simulated results are consistent with the experimental ones [38]. Disappeared GB1 lost ability to emit dislocations and is no longer a dislocation source.

Although GB2 and GB4 are the dislocation sources, the direction of uniaxial tension is parallel to GB1 and GB4, the loading direction is not beneficial to GB1 and GB4 to emit 
dislocations. The GB3 is far from local twin T1, the effect of GB3 on twin T1 is insignificant. Without interaction of lots of dislocations with twin, twin structures remain stable.

As tensile strain is up to $5.5 \%$, the detwin of twin T1 occurs, as shown in Figure 3. The grain G2 growths destroy twin T1. In the process of detwinning deformation, the stacking faults emitted from the twin boundary, $\mathrm{T} 1$, are observed. It demonstrated that the detwinning deformation is companied with dislocation activities, which is consistent with the simulation results given by $\mathrm{Li}$ et al. [7]. Recently $\mathrm{Ni}$ et al. [19] performed an experiment of $\mathrm{Ni}-\mathrm{Fe}$ alloy with preexisting growth twins to study the effect of grain size on deformation twining and detwinning. The experimental result revealed that the extensive detwinning occurred during the deformation in grains smaller than $35 \mathrm{~nm}$ and that new grains formed during grain growth did not contain twins. This suggests that the grain growth of nt metals may annihilate twin microstructure. $\mathrm{Ni}$ et al. experimental results support our simulation result.

Figure 4 displays curves of energy versus strain. The comparison of energy per atom both whole system and local region shows that the potential energy of local region decreases with tensile strain. As tensile loading increases, the energy of whole system increases, which is expected. As the energy of local region with twin microstructure decreases, the grain G2 grows with tensile deformation. The emitted partial dislocations from twin boundary interact with amorphous atoms of twin boundary, in which the amorphous atoms original from $\mathrm{HCP}$ atoms gradually become FCC atoms gradually, thereby reducing local energy. The variation of from a little high energy to lower energy for evolution of local microstructure is beneficial.

\subsection{The shear loading}

The same sample used in the tensile deformation is under

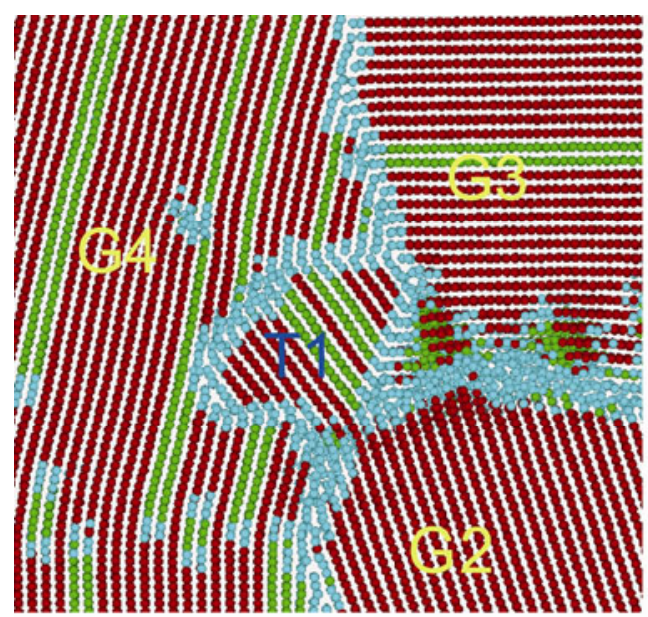

Figure 3 Atomic configuration of grain 1 and 4 during the tensile deformation, $\varepsilon_{y y}=5.5 \%$.

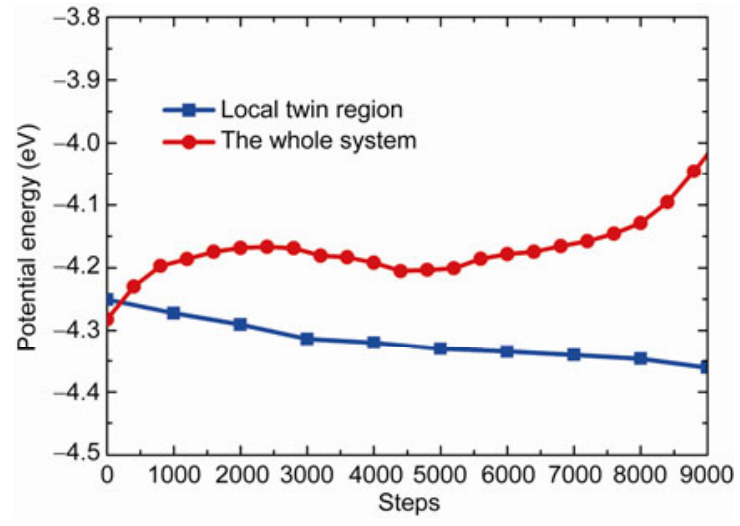

Figure 4 Comparison of system energy and local energy per atom.

the shear loading, the effect of different loading rates on the mechanical behavior of nt structure is investigated. In order to understand our simulation results clearly, the geometry parameter (area of nt microstructure) is taken as to describe variation of twin microstructure. Figure 5 shows the comparison of the area variation of circle twin $\mathrm{T} 1$ at four different loading rates, they are $1.5 \times 10^{9} \mathrm{~s}^{-1}, 3.1 \times 10^{9} \mathrm{~s}^{-1}, 7.7$ $\times 10^{9} \mathrm{~s}^{-1}$ and $1.2 \times 10^{10} \mathrm{~s}^{-1}$, respectively. From Figure 5, we know that the circle twin structure displays the stable state in the shear deformation process. At initial shear deformation, the area of nt $\mathrm{T} 1$ reduced greatly, meaning that $\mathrm{nt}$ $\mathrm{T} 1$ is sensitive to the shear deformation, it thus can contribute to the compression of the surrounding grain to nt T1. For the case of the shear strain rate $1.5 \times 10^{9} \mathrm{~s}^{-1}$, as the shear strain is about 0.025 , the area of twin T1 is quickly reduced, and it continues to reduce with the shear deformation, However, the reduced rate is slow, the area of nt T1 approaching a constant value. Thus we can conclude that under the shear deformation with the shear rate $1.5 \times 10^{9} \mathrm{~s}^{-1}$, twin $\mathrm{T} 1$ could experience a big deformation and still remains the circle twin structure. As the shear rate increases to $3.1 \times 10^{9} \mathrm{~s}^{-1}$ and $7.7 \times 10^{9} \mathrm{~s}^{-1}$, the same conclusion can be

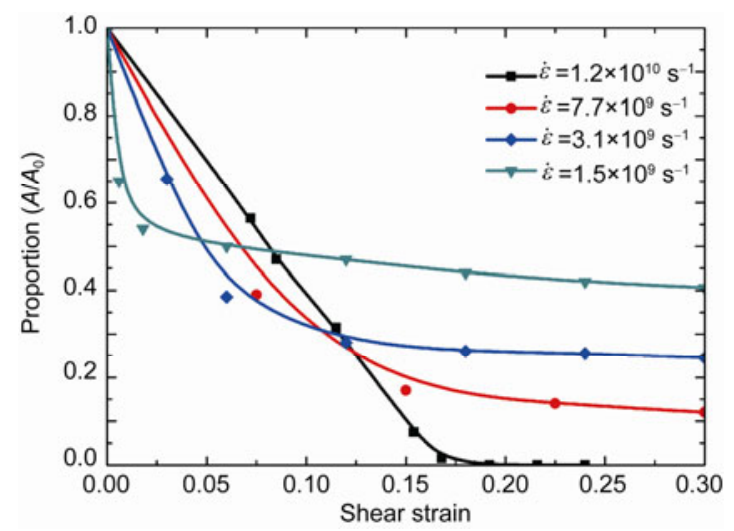

Figure 5 Variation of area of circle twin T1 during shear loading process at four different loading rates. 
obtained. As the shear strain is about 0.165 and 0.25 , the decline trend of area $\mathrm{T} 1$ begins to reduce gradually. The higher of the shear rate is, the smaller area of $\mathrm{T} 1$ is given. As the shear rate increases up to $1.2 \times 10^{10} \mathrm{~s}^{-1}$, the area of $\mathrm{T} 1$ decreases with deformation. As the shear strain is about 0.20 , the detwinning deformation occurs. Thus the circle twin $\mathrm{T} 1$ is destroyed. This may be a critical shear rate. As the shear rate is less than the critical value, it is the stable deformation of twin. If the shear rate is greater than the critical value, an unstable deformation of twin is observed, and detwinning occurs. Hodge et al. [20] examined $\mathrm{Cu}$ twin stability under four distinct mechanical loading states, tension, compression, tension-tension fatigue and torsion, the sample $\mathrm{Cu}$ contains parallel nanotwins about $40 \mathrm{~nm}$ thick. Experimental observations show that the column of twins in the $\mathrm{Cu}$ interior can persist up to the high shear strain of 1 under the shear deformation. Our simulations are in good agreement with experimental results.

Figures 6 and 7 display the atom configurations at the shear rate of $7.7 \times 10^{9} \mathrm{~s}^{-1}$ and $1.2 \times 10^{10} \mathrm{~s}^{-1}$, respectively. Figures 6(a) and 6(b) show that as the shear strain is up to $12.5 \%$ and $30 \%$, the twin structure is table, but the area of circle twin $\mathrm{T} 1$ decreases with the shear deformation. It can

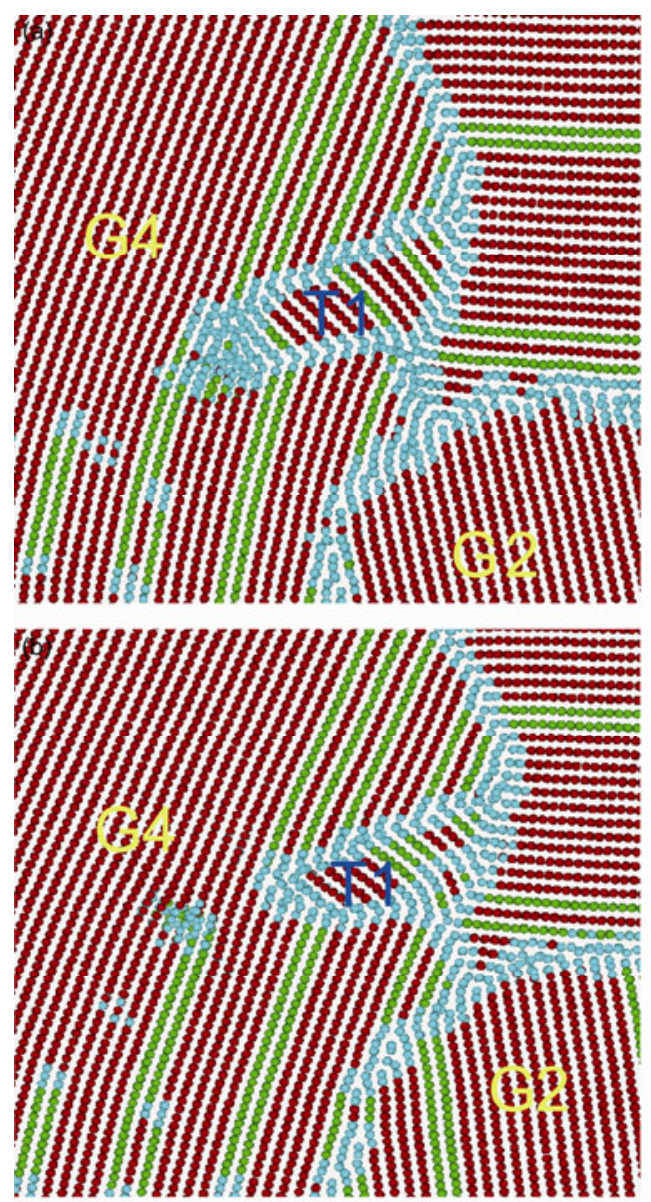

Figure 6 Local atomic configurations during the shear deformation at the rate of $\dot{\gamma}=7.7 \times 10^{9} \mathrm{~s}^{-1}$. (a) $\varepsilon_{y y}=12.5 \%$; (b) $\varepsilon_{y y}=30.0 \%$.
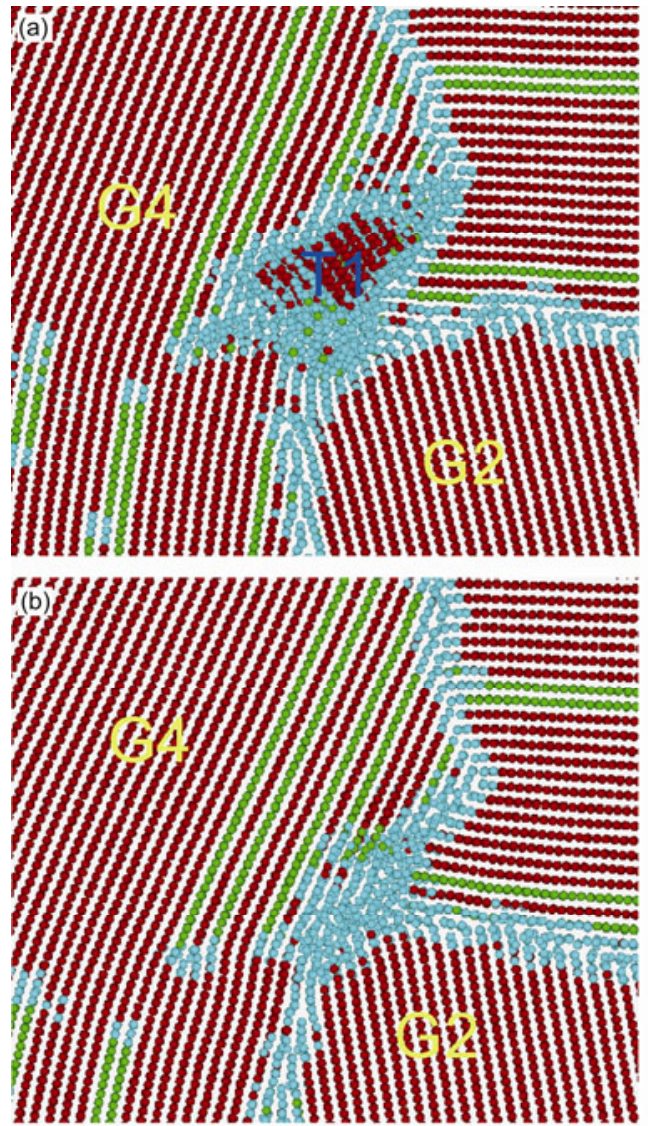

Figure 7 Local atomic configurations during the shear deformation at the rate of $\dot{\gamma}=1.2 \times 10^{10} \mathrm{~s}^{-1}$. (a) $\varepsilon_{y y}=12.5 \%$; (b) $\varepsilon_{y y}=20.0 \%$.

be noted that as the shear strain is up to $30 \%$, the shear stress is $0.5 \mathrm{GPa}$ (Figure 8) which is somewhat lower than the peak value $2.0 \mathrm{GPa}$, it is not possible to destroy the circle twin structure T1 in such low stress. The effect of the shear rate on twin deformation is observed in comparion Figure 6(a) with Figure 7(a), the area of circle twin T1 decreases with increasing the shear rate at the same shear strain. As the shear rate is up to $1.2 \times 10^{10} \mathrm{~s}^{-1}$, the detwinning deformation occurs. Figure 7 (b) shows that as the shear strain is up to $20 \%$, the circle twin structure is destroyed.

Figure 8 shows the curves of the shear stress versus strain, a comparison of the effect of two different shear rates on mechanical behavior is given. The peak of the stress at the shear rate of $1.2 \times 10^{10} \mathrm{~s}^{-1}$ is about $3.3 \mathrm{GPa}$, which is much higher than that at the shear rate of $7.7 \times 10^{9} \mathrm{~s}^{-1}$, which is about $2.0 \mathrm{GPa}$. It can be understood that under the high shear rate there is not enough sufficient time for a deformation system to relax, which leads to high stress and thus the twin T1 is destroyed. The results are consistent with the above analysis.

Figure 9 is the evolution of proportion of FCC, HCP, NON atoms versus time at the shear rate of $7.7 \times 10^{9} \mathrm{~s}^{-1}$ and $1.2 \times 10^{10} \mathrm{~s}^{-1}$, respectively. It can be used to understand the individual deformation mechanisms such as the dislocation mechanism, GB-associated mechanism, deformation twin- 


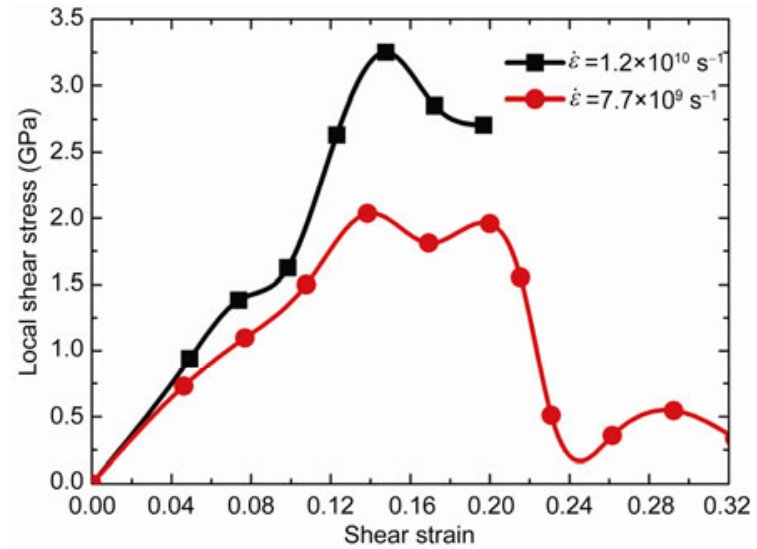

Figure 8 (Color online) Curves of local shear stress versus strain.
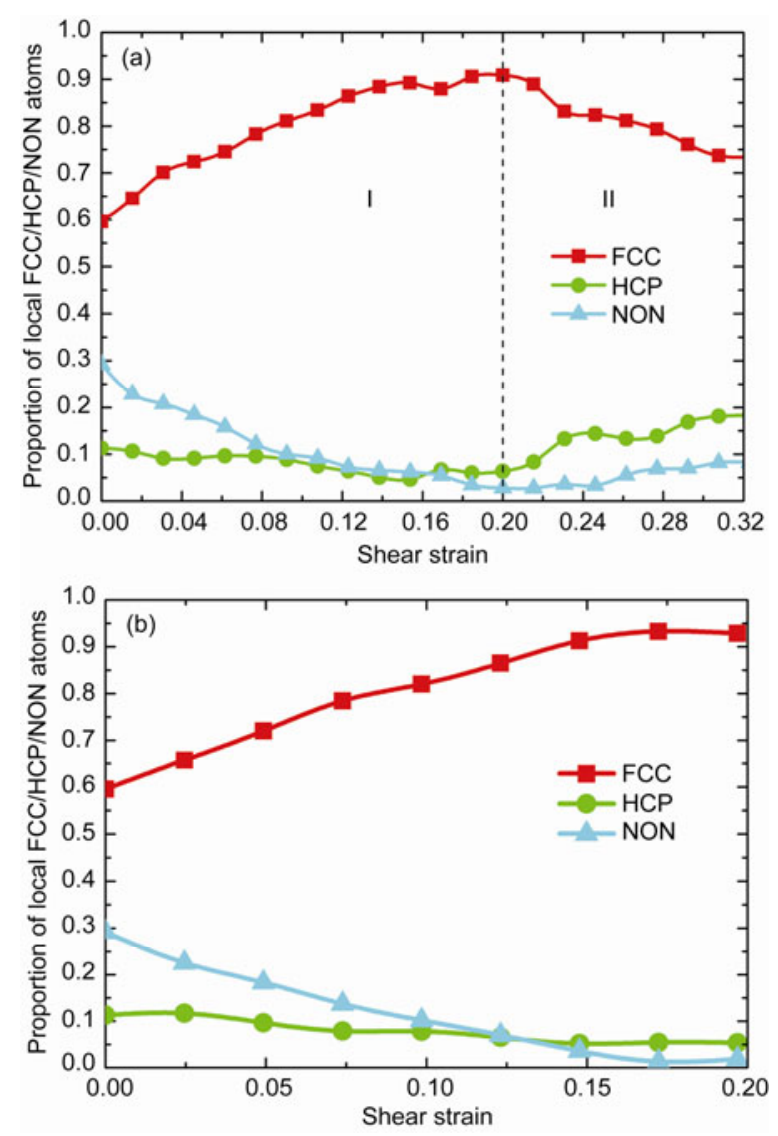

Figure 9 (Color online) Curves of atom proportion of $\mathrm{FCC} / \mathrm{HCP} / \mathrm{NON}$ atoms versus strain. (a) shear rate $\dot{\gamma}=7.7 \times 10^{9} \mathrm{~s}^{-1}$; (b) shear rate $\dot{\gamma}=1.2 \times 10^{10} \mathrm{~s}^{-1}$.

ning, and TB-associated mechanism work in parallel in the plastic deformation of the nt Ni sample during the shear loading. In zone I of Figure 9(a), an increase in FCC atoms accompanied with a decrease in HCP atoms indicates that plastic deformation is dominated by annihilation of partial dislocations and grain growth. Moreover, nt T1 is greatly reduced. Conversely, plastic deformation in zone II is dom- inated by dislocation nucleation and subsequent propagation, as HCP atoms and other atoms increase monotonously, and nt T1 is somewhat reduced. As the shear rate is $1.2 \times 10^{10} \mathrm{~s}^{-1}$, the different case is observed in Figure 9(b). A monotonously increase in FCC atoms accompanied with a decrease in HCP atoms and other atoms indicates that the plastic deformation is totally dominated by grain growth and nt T1 reduction, leading to detwinning.

\section{Conclusions}

In summary, MD simulations are carried out to understand the detwinning deformation of circle nt structure under tensile and shear loading. The simulation results show that nt structure is the stable during tensile and shear deformation. As deformation is large, the detwinning deformation occurs because of grain growth, which is consistent with experimental observation. Our simulation also shows that the detwinning deformation of shear loading is related to loading rate, as loading rate is sufficiently large, the detwinning deformation occurs.

This work was supported by the National Natural Science Foundation of China (Grant Nos. 11021262, 11172303 and 11132011) and National Basic Research Program of China (Grant No. 2012CB937500).

1 Lu K, Lu L, Suresh S. Strengthening materials by engineering coherent internal boundaries at the nanoscale. Science, 2009, 324: 349352

2 Dao M, Lu L, Shen Y F, et al. Strength, strain-rate sensitivity and ductility of copper with nanoscale twins. Acta Mater, 2006, 54: 5421-5432

3 Kumar K S, Van Swygenhoven H, Suresh S. Mechanical behavior of nanocrystalline metals and alloys. Acta Mater, 2003, 51: 5743-5774

4 Tang Q H, Wang T C, Shang B S, et al. Thermodynamic properties and constitutive relations of crystals at finite temperature. Sci China-Phys Mech Astron, 2012, 55: 918-926

5 Yang Z, Lu Z, Zhao Y P. Atomistic simulation on size-dependent yield strength and effects evolution of metal nanowires. Comp Maetr Sci. 2009, 46: 142-150

6 Zhu Y T, Liao X Z. Nanostructured metals - Retaining ductility. Nat Mater, 2004, 3: 351-352

7 Li X, Wei Y, Lu L, et al. Dislocation nucleation governed softening and maximum strength in nano-twinned metals. Nature, 2010, 464: 877-880

8 Schiotz J, Vegge T, Jacobsen K W. Atomic-scale modeling of the deformation of nanocrystalline metals. Mater Res Soc Sympos Proc, 1999, 583: 299-308

9 Shan Z, Stach E A, Wiezorek J M K, et al. Grain boundary-mediated plasticity in nanocrystalline nickel. Science, 2004, 305: 654-657

10 Zhou H F, Qu S X, Yang W. Toughening by nano-scaled twin boundaries in nanocrystals. Modell Simul Mater Sci Eng, 2010, 18: 065002

11 Christian J W, Mahajan S. Deformation twinning. Prog Mater Sci, 1995, 39: 1-157

12 Wu X L, Liao X Z, Srinivasan S G, et al. New deformation twinning mechanism generates zero macroscopic strain in nanocrystalline met- 
als. Phys Rev Lett, 2008, 100: 095701

13 Froseth A, Swygenhoven H V, Derlet P M. The influence of twins on the mechanical properties of nc-Al. Acta Mater, 2004, 52: 2259-2268

14 Ogata S, Li J, Yip S. Energy landscape of deformation twinning in bcc and fcc metals. Phys Rev B, 2005, 71: 224102

15 Li N, Wang J, Huang J Y, et al. Influence of slip transmission on the migration of incoherent twin boundaries in epitaxial nanotwinned $\mathrm{Cu}$. Scr Mater, 2011, 64: 149-152

16 Wang J, Li N, Anderoglu O, et al. Detwinning mechanisms for growth twins in face-centered cubic metals. Acta Mater, 2010, 58: 2262-2270

17 Wen H M, Zhao Y H, Li Y, et al. High-pressure torsion-induced grain growth and detwinning in cryomilled $\mathrm{Cu}$ powders. Philos Mag, 2010, 90: 4541-4550

18 Li L, Ungar T, Wang Y D, et al. Microstructure evolution during cold rolling in a nanocrystalline $\mathrm{Ni}-\mathrm{Fe}$ alloy determined by synchrotron X-ray diffraction. Acta Mater, 2009, 57: 4988-5000

19 Ni S, Wang Y B, Liao X Z, et al. Effect of grain size on the competition between twinning and detwinning in nanocrystalline metals. Phys Rev B, 2011, 84: 235401

20 Hodge A M, Furnish T A, Shute C J, et al. Twin stability in highly nanotwinned $\mathrm{Cu}$ under compression, torsion and tension. Scripta Mater, 2012, 66: 872-877

21 Foiles S M, Baskes M I, Daw M S. Embedded-Atom-Method Functions for the Fcc Metals $\mathrm{Cu}, \mathrm{Ag}, \mathrm{Au}, \mathrm{Ni}, \mathrm{Pd}, \mathrm{Pt}$, and Their Alloys. Phys Rev B, 1986, 33: 7983-7991

22 Tang Q H. Effect of size on mechanical behavior of Au pillars by molecuilar dynamics study. Sci China-Phys Mech Astron, 2012, 55: 1111-1117

23 Parrinello M, Rahman A. Polymorphic transitions in single-crystalsa new molecular-dynamics method. J Appl Phys, 1981, 52: 7182-7190

24 Tschopp M A, McDowell D L. Tension-compression asymmetry in homogeneous dislocation nucleation in single crystal copper. Appl Phys Lett, 2007, 90: 121916

25 Melchionna S, Ciccotti G, Holian B L. Hoover NPT dynamics for systems varying in shape and size. Mol Phys, 1993, 78: 533-544

26 Schiotz J, Tolla F D D, Jacobsen K W. Softening of nanocrystalline metals at very small grain sizes. Nature, 1998, 391: 561-563

27 Ma W, Zhu W J, Zhang Y L, et al. Construction of metallic nanocrystalline samples by molecular dynamics simulation. Acta Phys Sin, 2010, 59: 4781-4787

28 Yamakov V, Wolf D, Phillpot S R, et al. Deformation twinning in nanocrystalline Al by molecular dynamics simulation. Acta Mater, 2002, 50: 5005-5020

29 Qu S, Wang G, Zhou H, et al. Can nanoscale twin boundaries serve as dislocation sources in single crystals. Comput Mater Sci, 2011, 50: $1567-1570$

30 Nose S, Klein M L. Constant pressure molecular-dynamics for molecular-systems. Mol Phys, 1983, 50: 1055-1076

31 Hoover W G. Canonical dynamics-equilibrium phase-space distributions. Phys Rev A, 1985, 31: 1695-1697

32 Leach A R. Molecular Modelling: Principles and Applications. Harlow: Addison Wesley Longman, 1986. 355-359

33 Honeycutt J D, Andersen H C. Molecular dynamics study of melting and freezing of small Lennard-Jones clusters. J Phys Chem, 1987, 91: 4950-4963

34 Tsuzuki H, Branicio P S, Rino J P. Structural characterization of deformed crystals by analysis of common atomic neighborhood. Comp Phys Commun, 2007, 177: 518-523

35 Cao A J, Wei Y G. Formation of fivefold deformation twins in nanocrystalline face-centered-cubic copper based on molecular dynamics simulations. Appl Phys Lett, 2006, 89: 041919

36 Farnsworth H E, Haque C A, Zehner D M, et al. Influence of high temperature heat treatment on surface order and faceting of some Mo, W, Ta and Re crystal surface. Surf Sci, 1969, 17: 1-8

37 Huang W, Wang Y, Ge P. Meso-scale simulation on mechanical behavior of single crystal Titanium. Rare Met Mater Eng, 2010, 39: $469-472$

38 Akhtar A. Basal slip and twinning in alpha titanium single crystals. Metallur Trans, 1975, A6: 1105-1113 